\title{
Current Trends in the Incidence and Survival Rate of Urological Cancers in Korea
}

\author{
Jae Young Joung, MD, PhD \\ Jiwon Lim, MS ${ }^{2}$ \\ Chang-Mo Oh, MD, PhD2 \\ Kyu-Won Jung, MS² \\ Hyunsoon Cho, $\mathrm{PhD}^{2}$ \\ Sung Han Kim, MD, PhD ${ }^{1}$ \\ Ho Kyung Seo, MD, PhD' \\ Weon Seo Park, MD, PhD ${ }^{1}$ \\ Jinsoo Chung, $M D, P h D^{1}$ \\ Kang Hyun Lee, MD, $\mathrm{PhD}^{1}$ \\ Young-Joo Won, $\mathrm{PhD}^{2}$
}

${ }^{1}$ Center for Prostate Cancer,

National Cancer Center, Goyang,

${ }^{2}$ Cancer Registration and Statistics Branch,

National Cancer Center, Goyang, Korea
Correspondence: Kang Hyun Lee, MD, PhD

Center for Prostate Cancer,

National Cancer Center, 323 Ilsan-ro,

Ilsandong-gu, Goyang 10408, Korea

Tel: 82-31-920-1676

Fax: 82-31-920-1511

E-mail: uroonco@ncc.re.kr

Co-correspondence: Young-Joo Won, $\mathrm{PhD}$ Cancer Registration and Statistics Branch,

National Cancer Center, 323 Ilsan-ro,

Ilsandong-gu, Goyang 10408, Korea

Tel: 82-31-920-2015

Fax: 82-31-920-2179

E-mail: astra67@ncc.re.kr

Received March 30, 2016

Accepted September 11, 2016

Published Online September 23, 2016

\section{Purpose}

This descriptive study assessed the current trends in the incidence of urological cancers and patient survival in Korea.

\section{Materials and Methods}

In this nationwide retrospective observational study based on the data from the Korea National Cancer Incidence Database (KNCIDB), this study analyzed the age-standardized incidence rates (ASRs) and annual percentage changes (APCs) of kidney, bladder, prostate, testicular, and penile cancers as well as cancer of the renal pelvis and ureter between 1999 and 2012. The relative survival rates (RSRs) were calculated for urological cancer patients diagnosed between 1993 and 2012 from the KNCIDB data.

\section{Results}

Prostate cancer was diagnosed in 66,812 individuals followed by bladder $(41,549)$ and kidney $(36,836)$ cancers. The overall ASR $(18.26$ per 100,000$)$ increased with age because of the higher ASRs of bladder and prostate cancers in the elderly. The ASR for kidney cancer was highest in the 40-59-year-old group, whereas testicular cancer occurred most frequently before the age of 40 . The incidence of most urological cancers increased (overall APC, 6.39\%; $p$ < 0.001), except for penile (APC, -2.01\%; $p=0.05$ ) and bladder (APC, $-0.40 \%$; $\mathrm{p}=0.25$ ) cancers. The overall survival increased steadily (5-year RSR, 66.4\% in 1993-1995 vs. $84.2 \%$ in 2008-2012; p < 0.001), particularly for prostate (by $34.10 \%$ ) and kidney (by $16.30 \%$ ) cancers, but not for renal pelvis and ureter cancers $(-7.20 \%)$.

\section{Conclusion}

The most common urological cancer in Korea was prostate cancer followed by bladder and kidney cancers. The incidence of most urological cancers, except for penile and bladder cancers, increased. Survival also increased, particularly for prostate and kidney cancers.

Key words

Urologic neoplasms, Incidence, Survival, Korea 


\section{Introduction}

Cancer is a major public health problem worldwide [1,2]. In Korea, more than 200,000 new cancers are diagnosed each year, and cancer is responsible for one in four deaths [3]. In general, the incidence of urological cancers is higher in Western countries compared with that in Asian countries. For example, the incidence of prostate cancer is three to four times higher in Western countries than in Korea [4]. On the other hand, the rapidly increasing elderly population in eastern Asian countries, including Korea, might induce an increase in the incidence of urological cancers in the future. Over the last few years, the rates of prostate cancer in Korea appear to have risen steadily, with an up to $28.2 \%$ increase during 1996-1998 and 1999-2001 [5]. To the best of the authors' knowledge, there is limited data concerning the incidence of urological cancers in Korea. Furthermore, these data are not based on nationwide samples and concern specific cancer types such as prostate cancer $[5,6]$.

Improved knowledge regarding the incidence of urological cancers and patient survival rates can help assess diagnostic measures and the need for the continued promotion of urological cancer screening programs. We hypothesized that the overall incidence of urological cancer has increased in Korea, and that the patterns of each cancer have started to resemble those observed in Western countries. Using the Korea Central Cancer Registry (KCCR) data, this study aimed to assess trends in the incidence rate of urological cancers between 1999 and 2012 as well as the relative survival rate (RSR) of patients diagnosed with urological cancers from 1993 to 2012 in Korea.

\section{Materials and Methods}

\section{Data sources}

The KCCR was started as a nationwide, hospital-based cancer registry in 1980 by the Ministry of Health and Welfare and has covered the entire population since 1999. The Korea National Cancer Incidence Database (KNCIDB) is composed of the KCCR data and includes information on the cancer patient demographics, primary cancer site, morphology, diagnostic date, and initial treatment. For this study, the incidence and survival data were obtained from the KNCIDB. The urological cancer cases were classified according to the International Classification of Diseases for Oncology, third edition [7] and converted according to the International Classification of Diseases, 10th edition (ICD-10)
[8]. In this study kidney cancer (C64), bladder cancer (C67), prostate cancer (C61), cancer of the renal pelvis or ureter (C65, C66), testis cancer (C62), and penile cancer (C60) were included as urological cancers.

The Institutional Review Board of the National Cancer Center approved this study (IRB No. NCC2015-0249). The ethics committee waived the need for participants' consent because this study employed collected data that were anonymized for statistical analysis.

\section{Analysis}

The age-standardized incidence rates (ASRs) were calculated for each cancer site in patients diagnosed with cancer between 1999 and 2012 according to sex and age group $(<40$, $40-59,60-69$, and $\geq 70$ years) using the world standard population. ASRs were expressed per 100,000 persons. The incidence trends for urological cancers were analyzed by the annual percentage change (APC) for each sex from 1999 to 2012. The male/female ratio was calculated as the ratio of the ASR in men to that in women.

The survival data were retrieved using the KNCIDB for individuals newly diagnosed with cancer from 1993 to 2012 and followed until December 31, 2013. The 5-year RSRs were calculated according to 5 periods of diagnosis (1993-1995, 1996-2000, 2001-2005, 2006-2010, and 2008-2012). The RSRs were calculated by comparing the observed survival with the expected survival using the Ederer II method. All analyses were conducted using the SAS ver. 9.2 (SAS Institute Inc., Cary, NC), and $p<0.05$ was considered statistically significant.

\section{Results}

\section{Incidence}

During 1999-2012, urological cancer occurred in 155,991 patients (Table 1). The most common cancer was prostate cancer (66,812 patients) followed by bladder cancer $(41,549$ patients), kidney cancer (36,836 patients), renal pelvis and ureter cancer ( 7,537 patients), testis cancer (2,439 patients), and penile cancer ( 818 patients). The overall ASR for urological cancer was 18.26 per 100,000 persons. The ASR was 37.00 for men and 4.72 for women. The higher ASR observed among men was due most likely to prostate cancer. On the other hand, a similar difference between sexes was also found for bladder and kidney cancer.

Table 2 shows the ASRs per 100,000 persons by age group. The overall ASR of urological cancer increased with age 

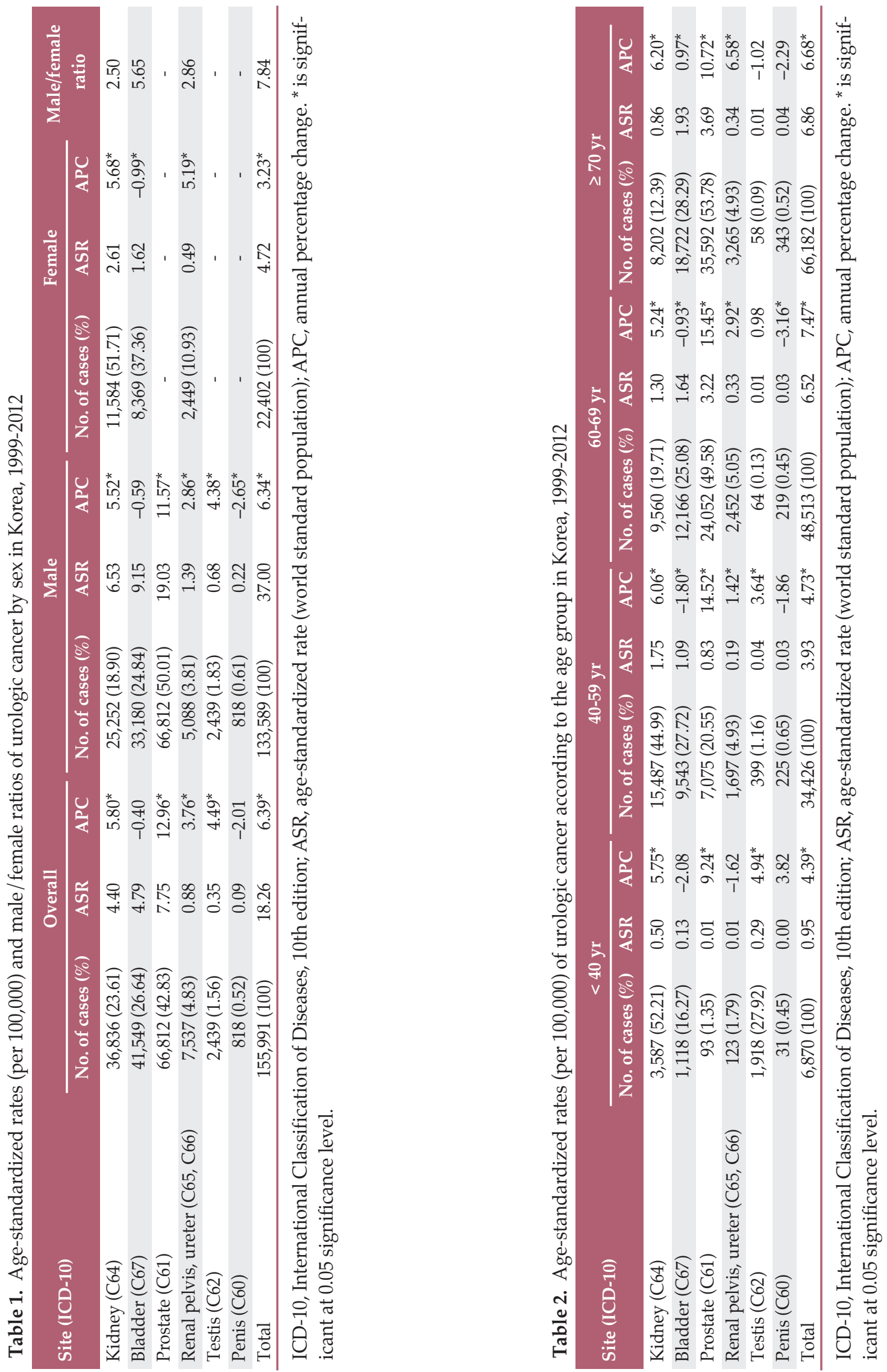

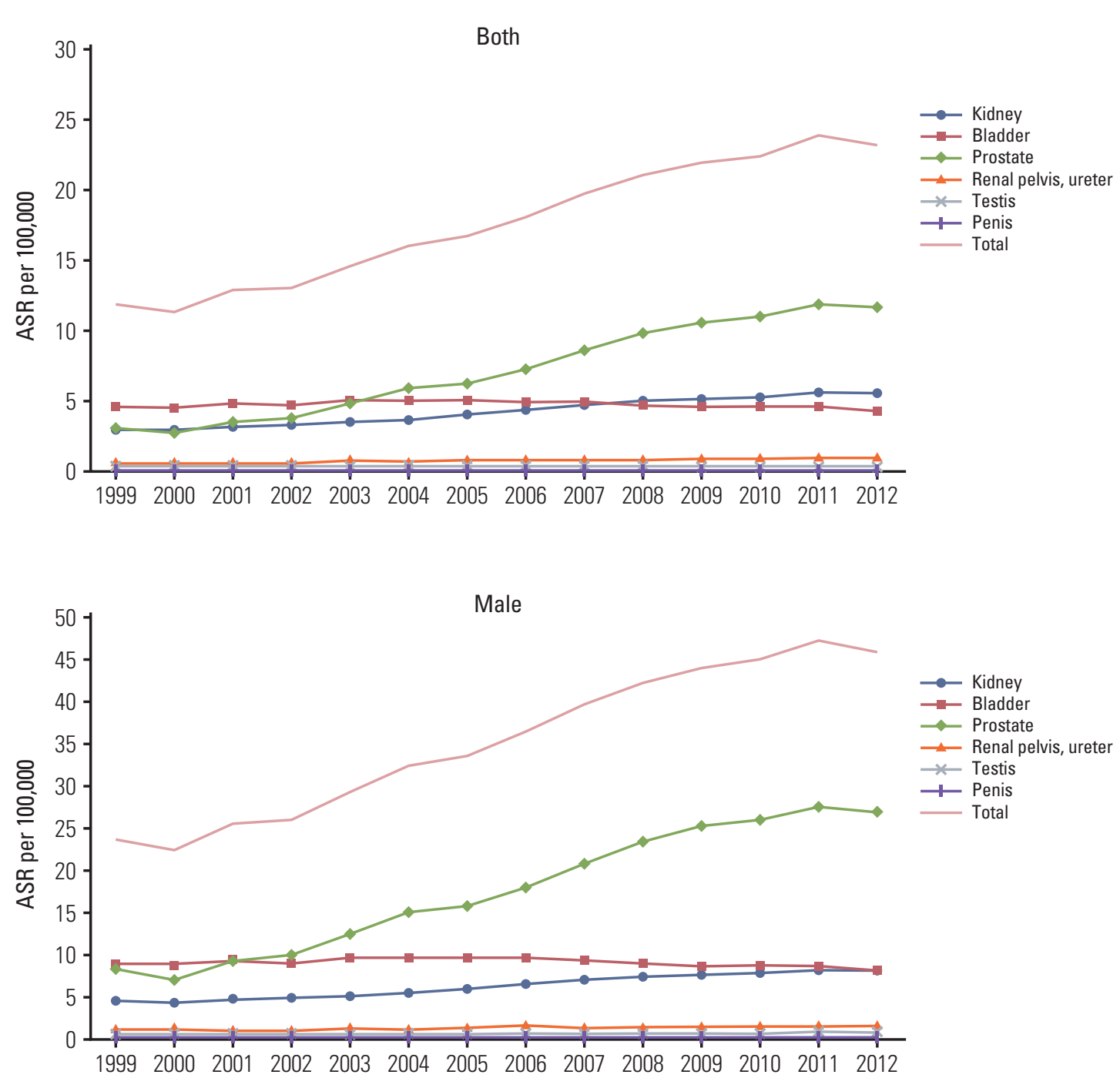

C

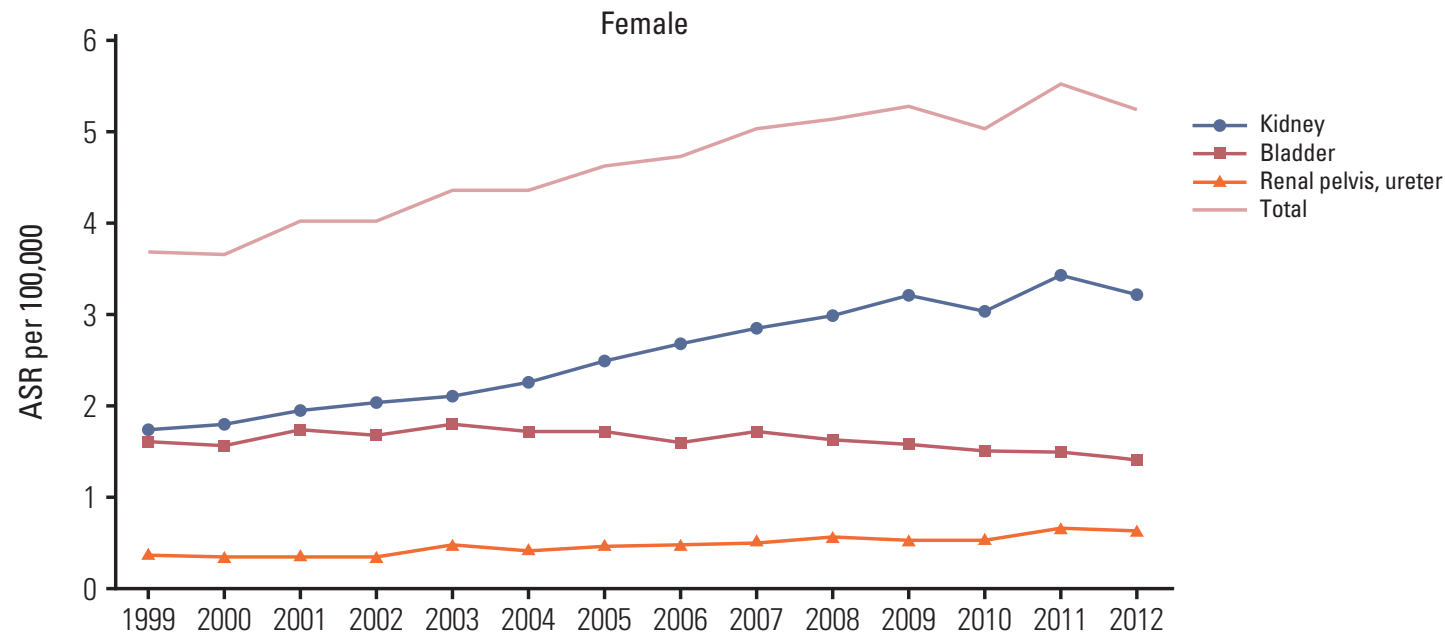

Fig. 1. The change of age-standardized incidence rate (ASR) of urologic cancer per 100,000 populations, 1999-2012. (A) Both. (B) Male. (C) Female. 
because of the higher ASR of bladder cancer and prostate cancer among the elderly. However, kidney cancer showed the highest ASR among those 40-59 years old (ASR, 1.75) while the ASR of testis cancer was the highest among those $<40$ years old (ASR, 0.29).

Fig. 1A shows the changes in ASRs for urological cancers in Korea from 1999 to 2012. The overall ASR for all cancers increased with time. This was influenced most likely by a gradual increase in the ASR for prostate cancer. Kidney cancer and testis cancer also showed a steady increase but the ASR for bladder cancer and cancer of the renal pelvis and ureter did not increase. In particular, the ASR of bladder cancer decreased since 2007. When examined by sex, the ASR change for all cancers in men showed a similar pattern with that in the total population (Fig. 1B). However, among women, the ASR for kidney cancer and cancer of the renal pelvis and ureter steadily increased while the ASR for bladder cancer decreased (Fig. 1C).

The overall APC for all urological cancers between 1999 and 2012 was $6.39 \%$ of the total population $(\mathrm{p}<0.05)$ (Table 1$)$. In contrast, the incidence of penile cancer decreased with an overall APC of $-2.01 \%(\mathrm{p}<0.05)$. The incidence of bladder cancer also decreased with an overall APC of $-0.40 \%$, but this was not statistically significant ( $\mathrm{p}=0.25)$. The APC of kidney cancer was similar between men and women while the APC of renal pelvis and ureter cancer was higher among women than men (Fig. 2). The APC of prostate cancer was highest among those $60-69$ years old $(15.45 \%)$ followed by those 40-59 years old (14.52\%) (Table 2$)$.

\section{Survival}

Table 3 shows the 5-year RSR for urological cancers between 1993 and 2012. The overall 5-year RSR for all urological cancers was $78.6 \%$ during this period. From 1993 to 2012, the survival increased steadily from $66.4 \%$ in 1993-1995 to $84.2 \%$ in 2008-2012, an increase of $17.8 \%$ ( $p<0.05$ ). Remarkably, prostate cancer survival also increased from $58.2 \%$ in $1993-1995$ to $92.3 \%$ in 2008-2012, an increase of $34.1 \%$. An increase in the 5-year RSR was also observed for kidney cancer $(16.3 \%)$. On the contrary, the 5-year RSR of renal pelvis and ureter cancers decreased $(-7.2 \%)$. When examined according to age group, the 5-year RSR of most urological cancers was longer in the younger age groups than the older age groups. However, prostate cancer patients $<40$ years old had the worst 5-year RSR (44.3\%) (Table 4). The 10year RSRs showed similar patterns (S1 Table).

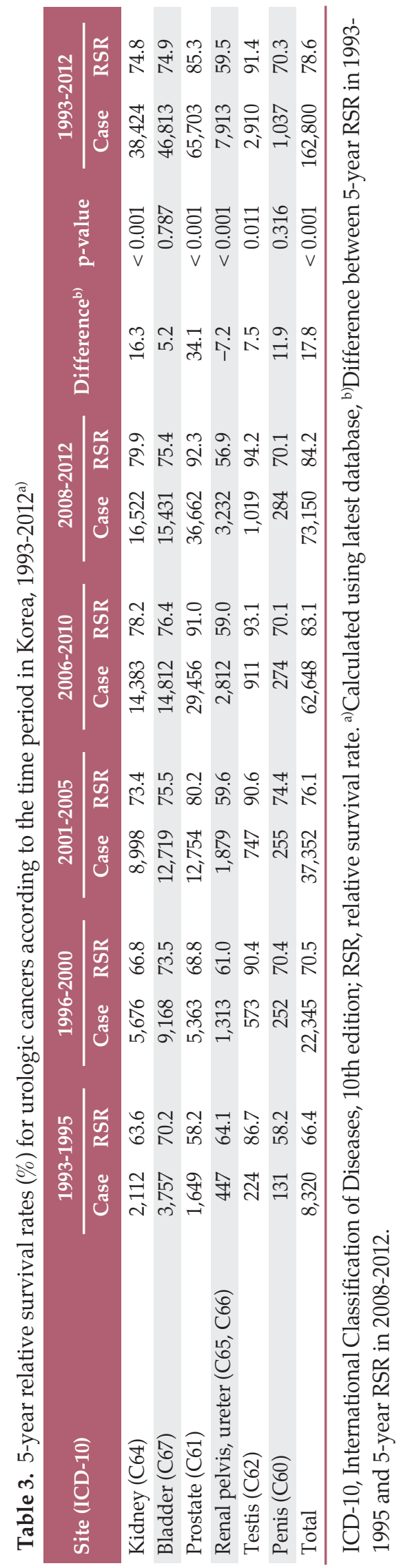

VOLUME 49 NUMBER 3 JULY 2017 


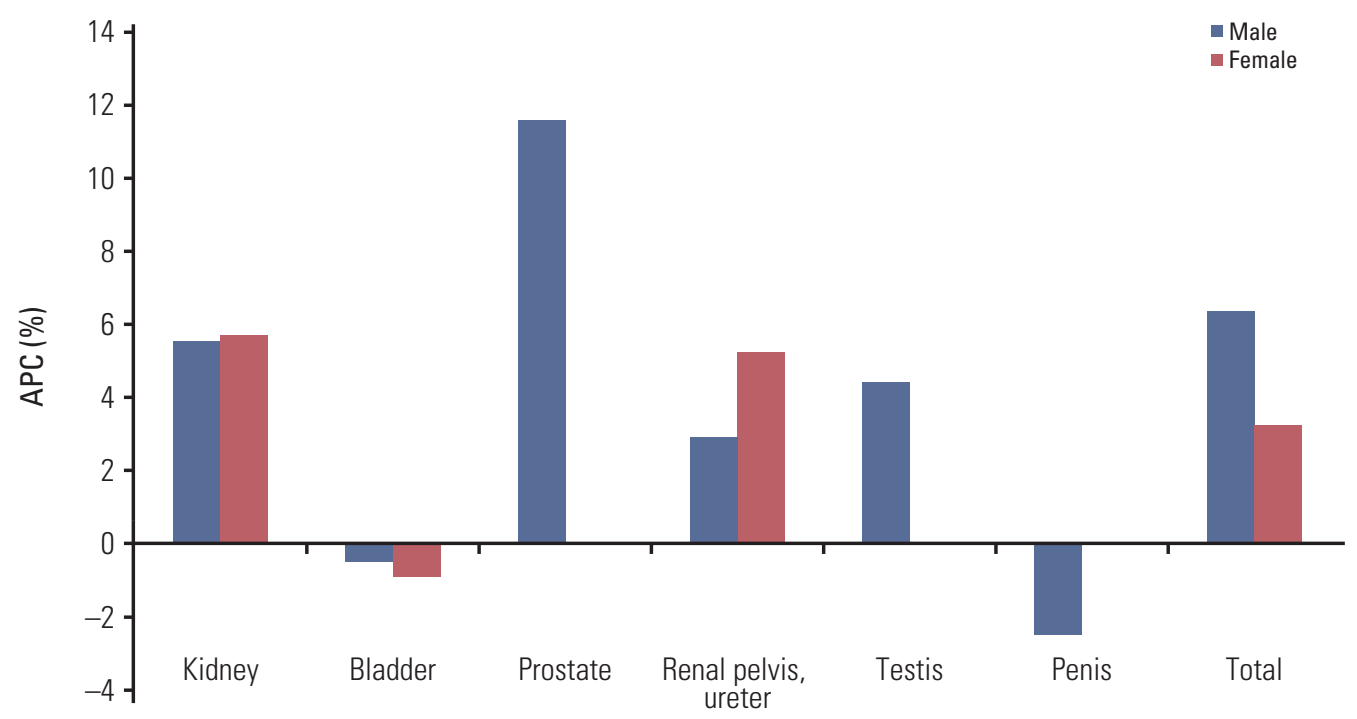

Fig. 2. Annual percentage change (APC) of urologic cancer by sex in Korea, 1999-2012.

Table 4. Five-year relative survival rate (\%) of urologic cancer according to the age group in Korea, 1993 to 2012

\begin{tabular}{lllll} 
Site (ICD-10) & $<40$ & $40-59$ & $60-69$ & $\geq 70$ \\
Kidney (C64) & 87.5 & 81.4 & 71.2 & 55.5 \\
Bladder (C67) & 91.6 & 85.6 & 79.0 & 62.5 \\
Prostate (C61) & 44.3 & 86.1 & 88.7 & 82.7 \\
Renal pelvis, ureter (C65, C66) & 78.9 & 67.1 & 62.6 & 50.0 \\
Testis (C62) & 93.3 & 88.4 & 76.5 & 45.1 \\
Penis (C60) & 75.8 & 69.0 & 71.7 & 69.7 \\
Total & 89.1 & 82.9 & 81.1 & 71.7 \\
\hline
\end{tabular}

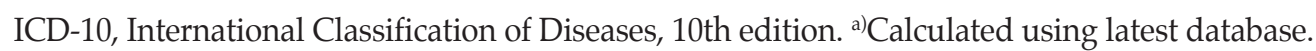

\section{Discussion}

In Korea, cancer registration began officially in 1980 with the formation of the KCCR, and reporting of cancer cases has been fairly complete since 1999 [9]. Analyzing the incidence of urological cancer may assist in its early detection and prevention as well as promote a better understanding of the urological cancer patterns in Korea.

In 2002, the Korean Urological Cancer Society examined the incidence of urological cancer in Korea based on the survey data of 46 hospitals between 1985 and 1999. During that period, bladder cancer was the most common urological cancer; the incidence of prostate cancer and renal cancer was similar, and male patients outnumbered female patients by a ratio of 5.4:1 [6]. Recent reports concerning the incidence of urologic cancers, including kidney, bladder, and prostate cancer, between 1999 and 2011 revealed prostate cancer to be the most common urological cancer, which are similar to those of the current study [10].

\section{Prostate cancer}

Generally, prostate cancer is not a common cancer in Asian countries, including Korea, compared to Western countries (Fig. 1, S2 Table); however, the incidence of prostate cancer has begun to increase recently $[3,5,6]$. Interestingly, Western countries have higher incidence rates of prostate cancer than Asian countries. On the other hand, the incidence of prostate cancer differ according to the socioeconomic status across 
Asian countries. High-income Asian countries, including Korea, Japan, and Singapore, have higher incidences of prostate cancer than low-income Asian countries, such as Philippines, Malaysia, and Thailand (S3 Fig.). Increasing age, high dietary fat intake, and cigarette smoking, as well as predisposing genetic factors, are considered common risk factors $[11,12]$. The rapid increase in the incidence of prostate cancer may be due to the widespread use of a prostate-specific antigen (PSA), which also induced stage migration of newly diagnosed prostate cancers to localized disease at diagnosis. For example, a previous study including 46 hospitals found that prostate cancer had been diagnosed in 2,417 patients comprising $18.3 \%$ of all urological cancers cases during 1985-1999 [6]. In another study, although the incidence of prostate cancer was low (7.9 per 100,000 man-years), it increased by $28.2 \%$ between 1996-1998 and 1999-2001 [5]. Furthermore, in the present study, 66,812 patients in the KCCR were diagnosed with prostate cancer between 1999 and 2012, a much higher number than previous studies examining survey data [6].

Based on the KCCR annual report, prostate cancer comprised $4.1 \%$ of all newly diagnosed cancers in 2012 with a crude incidence rate of 18.4 per 100,000 persons and an ASR of 11.6 per 100,000 persons, making prostate cancer the seventh most common cancer among the total population and the fifth most common cancer among men [3]. The ASR for prostate cancer was highest among those $\geq 70$ years old, but the APC for prostate cancer was highest among those 60-69 years old $(15.4 \%)$. These results are in accordance with general knowledge about prostate cancer development. In addition, the APC among those 40-59 years old was also high $(14.5 \%)$ and comparable to the rate among those in their 60 s. Considering the longer estimated survival observed in the younger age group, there should be more concern about a prostate cancer diagnosis for those in their 50s and 60s. In addition, the APC of prostate cancer among the total Korean population was 12.96 between 1999 and 2012, an APC that is much higher than in Japan (4.8) between 1985 and 2000 [13]. On the other hand, prostate cancer survival has increased remarkably, which may be related to the PSA test commonly used since the late 1990s that resulted in an increased rate of localized prostate cancer among newly diagnosed prostate cancer patients. However, the role of PSA-based screening in a prostate cancer diagnosis is still controversial because screening implementation has not decreased the prostate cancer mortality significantly [14]. In Korea, prostate cancer is not included in the national cancer screening program. Prostate cancer in Korean men has different biological characteristics than in Western populations. For Korean men, the incidence of high-grade or advanced-stage prostate cancer is higher $[15,16]$, even when the levels of PSA are low, which may be due to late detection or differences specific to the
Asian population. This emphasizes the need to detect prostate cancer at its early stages in Korean men.

\section{Bladder cancer}

The ASR of bladder cancer was similar in Japan and Korea (5.6 and 5.2, respectively) (S2 Table). On the other hand, despite the lower incidence of bladder cancer in Korea than in Western countries, bladder cancer remained the most common urological cancer in Korea before 2004. In addition, the incidence of bladder cancer gradually increased until 2003 and generally affected those $\geq 50$ years old, peaking among those older than 70 .

In the United States, the incidence of bladder cancer is increasing among men and declining slightly among women. In Japan, the incidence rate has slowly increased with an APC of 0.9 between 1985 and 2003 [13]. Therefore, compared to the APC of other urological cancers, including prostate and kidney cancer, the change in bladder cancer was not remarkable in Korea. In general, bladder cancer is prevalent in men, and in this study, the male to female ASR ratio was 5.68 between 1999 and 2012, which is similar to a previous study (ASR, 5.50 between 1985 and 1999) [6]. Since 2008, the ASR of bladder cancer has decreased, and in 2012, the ASR was 4.30 , which is the lowest value compared to other urological cancers (prostate cancer, 11.65; kidney cancer, 5.60). This may be related to cigarette smoking, which is considered the most important risk factor in bladder cancer [17]. For example, cigarette smoking increased rapidly in Korean society until the 1990s. However, since 1999, the rate of cigarette smoking in Korean adults has decreased gradually, which might be related to the decreasing incidence of bladder cancer.

\section{Kidney cancer}

The incidence of kidney cancer is higher in North America and Europe than in Asia and South America [18]. A previous report showed that male patients outnumbered female patients by a ratio of 2.3:1 between 1985 and 1999 [6]. In the United States, the ASR increased from 10.6 in 2001 to 12.4 in 2010, and the incidence in men was almost double that in women [19]. As shown in S2 Table, most Western countries have a higher ASR than Asian countries, such as Korea and Japan. Interestingly, Korea tended to have a higher ASR of kidney cancer than Japan among both men and women in 2012. In Japan, the APC in kidney cancer was 4.8 between 1985 and 1996 based on population-based data [13]. In Korea, the incidence of kidney cancer increased steadily during the study period (APC, 5.80). This increase may be related to early or incidentally detected kidney cancer derived from the prevalent health check-ups, including radiologic examina- 
tions, such as ultrasonography and computed tomography, since the 1990s in Korea. In addition, kidney cancer showed the highest ASR (1.75) in the 40-59 years age group, the period when Korean adults usually undergo health screening supported by the National Health Insurance system for the first time in their life. Accordingly, the survival of kidney cancer is currently increasing in Korea due to incidental and early detection of kidney cancer.

On the contrary, when compared with other urological cancers that usually develop in old age, kidney cancer is more prevalent in the middle-aged group, and the highest ASR is found among those aged 40-59 years. In the United States, however, kidney cancer is the most prevalent among an even younger age group (20 to 39 years old) [19]. This may be because of the association between renal cell carcinoma and obesity [20]. Therefore, since obesity in younger age groups has been increasing for several decades in the United States, childhood obesity may be at least partially responsible for the higher APC in 20-39 years age group. Furthermore, childhood obesity is currently an important health issue in Korea because of the high fat diet and Westernized food intake. In Korea, the prevalence of obesity has increased markedly since 1998 [21], indicating that the incidence of kidney cancer may increase in the future.

\section{Testis and penile cancer}

Testicular cancer is rare urological cancers; however, the incidence rate has been steadily increasing in Western countries, which is more common than Asian countries, including Korea. The incidence of testicular cancer in Western countries is approximately 5-9 times of that in Korea [4]. In Korean men, middle-aged men had the highest incidence of testicular cancer because of the commonly diagnosed seminoma. In general, testicular cancer is largely a disease of young and middle-aged men, but approximately $7 \%$ of cases occur in children and teens, and approximately $7 \%$ of cases occur in men over the age of 55 [22].

Penile cancer occurs in approximately one of 100,000 men in the United States [23]. Although penile cancer is more common in some parts of Asia, it is rare among Koreans and Israeli Jews (ASR, 0.22 in Koreans and 0.10 in Israeli Jews), which might be related to improvements in hygiene or commonly performed circumcisions $[24,25]$.

Despite the use of a population-based registry, there were some limitations to this study. First, because the KCCR does not collect data regarding the tumor characteristics, such as tumor stage, histological grade, or specific treatments, we could only examine relative survival and not cancer-specific survival or overall survival. Moreover, the KCCR does not record the cancer risk factors, such as tobacco/alcohol use, exposure to carcinogenic arsenic, dietary habits, and lifestyle, and this lack of data makes it more difficult to determine the real relationship between these etiological factors and the urinary tract cancer risk. Second, because of the very low incidence in Korea, some urological cancers, such as testicular cancer and penile cancer, were not analyzed comprehensively. Third, a study of cancer trends is mainly affected by the completeness of cancer registration. The completeness of cancer registration in the early period might be lower than that in recent years. These differences in completeness according to the time period might cause an overestimation or underestimation of the cancer trends. In addition, many Korean insurance companies do not currently cover patients with Ta bladder tumors or carcinoma in-situ (CIS) lesions. Therefore, several cases of Ta tumors or CIS lesions may not have been counted as new instances of cancer, which may have contributed to the apparent decrease in the incidence of bladder and renal pelvis or ureter tumors. Finally, this study was conducted in the Korean population, which consists almost entirely of ethnic Koreans, whereas the Korean health care system is markedly different from those of other countries. These factors should be taken into consideration when interpreting the present findings. Despite these shortcomings, this nationwide registry-based study included the largest numbers of cases of these rare diseases and contained information that is more than $97 \%$ complete and encompasses entire South Korea. Therefore, the results are likely to be the most accurate and can allow for an analysis of the internal validity and generalizability.

\section{Conclusion}

In Korea, prostate cancer was the most common urological cancer followed by bladder cancer and kidney cancer. The ASR of urological cancer was 18.26 per 100,000 persons, and men had a higher incidence than women. The incidence of urological cancers generally increases with age; however, kidney cancer and testicular cancer were diagnosed more frequently in the younger age groups. In addition, the urological cancer survival rates are currently increasing. In particular, the 5-year RSR for prostate cancer and kidney cancer has increased remarkably.

\section{Electronic Supplementary Material}

Supplementary materials are available at Cancer Research and Treatment website (http://www.e-crt.org). 


\section{Conflicts of Interest}

Conflict of interest relevant to this article was not reported.

\section{Acknowledgments}

This work was supported by a research grant from the National Cancer Center (No. 1310220), Republic of Korea.

\section{References}

1. Shin HR. Global activity of cancer registries and cancer control and cancer incidence statistics in Korea. J Prev Med Public Health. 2008;41:84-91.

2. Torre LA, Bray F, Siegel RL, Ferlay J, Lortet-Tieulent J, Jemal A. Global cancer statistics, 2012. CA Cancer J Clin. 2015;65:87108.

3. Jung KW, Won YJ, Kong HJ, Oh CM, Cho H, Lee DH, et al. Cancer statistics in Korea: incidence, mortality, survival, and prevalence in 2012. Cancer Res Treat. 2015;47:127-41.

4. GLOBOCAN 2012: estimated cancer incidence, mortality and prevalence worldwide in 2012. All cancers (excluding nonmelanoma skin cancer) estimated incidence, mortality and prevalence worldwide in 2012 [Internet]. Geneva: World Health Organization; 2016 [cited 2016 Mar 1]. Available from: http://globocan.iarc.fr/Pages/fact_sheets_cancer.aspx\#.

5. Park SK, Sakoda LC, Kang D, Chokkalingam AP, Lee E, Shin $\mathrm{HR}$, et al. Rising prostate cancer rates in South Korea. Prostate. 2006;66:1285-91.

6. Cheon J, Kim CS, Lee ES, Hong SJ, Cho YH, Shin EC, et al. Survey of incidence of urological cancer in South Korea: a 15-year summary. Int J Urol. 2002;9:445-54.

7. Fritz A, Percy C, Jack A, Shanmugaratnam K, Sobin L, Parkin $\mathrm{DM}$, et al. International classification of diseases for oncology. 3rd ed. Geneva: World Health Organization; 2000.

8. World Health Organization. International statistical classification of diseases and related health problems. 10th rev. Geneva: World Health Organization; 1994.

9. Shin HR, Won YJ, Jung KW, Kong HJ, Yim SH, Lee JK, et al. Nationwide cancer incidence in Korea, 1999 2001; first result using the national cancer incidence database. Cancer Res Treat. 2005;37:325-31.

10. Song W, Jeon HG. Incidence of kidney, bladder, and prostate cancers in Korea: an update. Korean J Urol. 2015;56:422-8.

11. Huncharek M, Haddock KS, Reid R, Kupelnick B. Smoking as a risk factor for prostate cancer: a meta-analysis of 24 prospective cohort studies. Am J Public Health. 2010;100:693-701.

12. Giovannucci E, Rimm EB, Colditz GA, Stampfer MJ, Ascherio A, Chute CG, et al. A prospective study of dietary fat and risk of prostate cancer. J Natl Cancer Inst. 1993;85:1571-9.

13. Katanoda K, Hori M, Matsuda T, Shibata A, Nishino Y, Hattori $\mathrm{M}$, et al. An updated report on the trends in cancer incidence and mortality in Japan, 1958-2013. Jpn J Clin Oncol. 2015;45:
390-401.

14. Andriole GL, Crawford ED, Grubb RL 3rd, Buys SS, Chia D, Church TR, et al. Prostate cancer screening in the randomized Prostate, Lung, Colorectal, and Ovarian Cancer Screening Trial: mortality results after 13 years of follow-up. J Natl Cancer Inst. 2012;104:125-32.

15. Choi SK, Song C, Shim M, Min GE, Park J, Jeong IG, et al. Prevalence of high-grade or insignificant prostate cancer in Korean men with prostate-specific antigen levels of 3.0-4.0 ng/mL. Urology. 2015;85:610-5.

16. Jeong IG, Dajani D, Verghese M, Hwang J, Cho YM, Hong JH, et al. Differences in the aggressiveness of prostate cancer among Korean, Caucasian, and African American men: A retrospective cohort study of radical prostatectomy. Urol Oncol. 2016;34:3.e9-14.

17. Morrison AS, Buring JE, Verhoek WG, Aoki K, Leck I, Ohno $\mathrm{Y}$, et al. An international study of smoking and bladder cancer. J Urol. 1984;131:650-4.

18. Chow WH, Dong LM, Devesa SS. Epidemiology and risk factors for kidney cancer. Nat Rev Urol. 2010;7:245-57.

19. King SC, Pollack LA, Li J, King JB, Master VA. Continued increase in incidence of renal cell carcinoma, especially in young patients and high grade disease: United States 2001 to 2010. J Urol. 2014;191:1665-70.

20. Adams KF, Leitzmann MF, Albanes D, Kipnis V, Moore SC, Schatzkin A, et al. Body size and renal cell cancer incidence in a large US cohort study. Am J Epidemiol. 2008;168:268-77.

21. Park HS, Park CY, Oh SW, Yoo HJ. Prevalence of obesity and metabolic syndrome in Korean adults. Obes Rev. 2008;9: 104-7.

22. Pike MC, Chilvers CE, Bobrow LG. Classification of testicular cancer in incidence and mortality statistics. Br J Cancer. 1987;56:83-5.

23. Bleeker MC, Heideman DA, Snijders PJ, Horenblas S, Dillner J, Meijer CJ. Penile cancer: epidemiology, pathogenesis and prevention. World J Urol. 2009;27:141-50.

24. Larke NL, Thomas SL, dos Santos Silva I, Weiss HA. Male circumcision and penile cancer: a systematic review and metaanalysis. Cancer Causes Control. 2011;22:1097-110.

25. Minhas S, Manseck A, Watya S, Hegarty PK. Penile cancer-prevention and premalignant conditions. Urology. 2010;76 (2 Suppl 1):S24-35. 